\title{
A Novel Graph-Based Suboptimal Multiuser Detector for FDM-CPM Transmissions
}

\author{
Amina Piemontese, Student Member, IEEE, and Giulio Colavolpe, Member, IEEE
}

\begin{abstract}
We consider a frequency division multiplexed (FDM) system where each user employs a continuous phase modulation (CPM), serially concatenated with an outer code through an interleaver, and iterative detection/decoding. In such a system, the spectral efficiency can be increased by reducing the spacing between two adjacent channels, thus increasing the relevant interference. Hence, we address the design of lowcomplexity suboptimal multiuser detectors able to effectively cope with such an interference. We extend some well known multiuser detection algorithms proposed for code division multiple access (CDMA) systems. Moreover, we introduce a new detection scheme using the framework based on factor graphs (FGs) and the sum-product algorithm (SPA). The simulation results show that the described algorithms allow to effectively reduce the spacing, thus increasing the spectral efficiency, and in particular, the proposed detection scheme results to be the most effective one in terms of performance and computational complexity.
\end{abstract}

Index Terms-Continuous phase modulations, frequency division multiplexing, iterative detection and decoding, multiuser detection.

\section{INTRODUCTION}

$\mathbf{I}$ $\mathrm{N}$ frequency division multiplexed (FDM) systems, the spectral efficiency can be increased by reducing the spacing between two adjacent channels, thus allowing overlap in frequency and hence admitting a certain amount of interference. This aspect has been investigated from an informationtheoretic point of view for linear [1] as well as continuous phase modulations (CPMs) [2], showing that a significant improvement can be obtained through packing even when at the receiver side a single-user detector is employed. ${ }^{1}$ When a multiuser receiver is adopted, the benefits in terms of spectral efficiency can be even larger and the signals can be packed denser and denser [1], [2].

Since, as known, the complexity of the optimal multiuser detector increases exponentially with the number of channels, suboptimal detection schemes are required. In the case of a satellite FDM system using linear modulations, the adoption of reduced-complexity multiuser detection (MUD) algorithms

Manuscript received July 20, 2009; revised February 11, May 12, and July 2, 2010; accepted July 2, 2010. The associate editor coordinating the review of this paper and approving it for publication was K. S. Kim.

The paper was presented in part at the IEEE International Symposium on Information Theory (ISIT'09), Seoul, Korea, June 2009. This work is funded by Newtec Cy, Sint-Niklaas, Belgium.

The authors are with the Università di Parma, Dipartimento di Ingegneria dell'Informazione, Viale G. P. Usberti, 181A, I-43100 Parma, Italy (e-mail: piemontese@tlc.unipr.it, giulio@unipr.it).

Digital Object Identifier 10.1109/TWC.2010.071410.091078

${ }^{1}$ In this context, the terms "single-channel" and "multi-channel" would be more suitable than "single-user" and "multiuser". However, in this paper we will use the terms "users" and "channels" interchangeably. borrowed from the literature on code division multiple access (CDMA) is investigated in [1], [3] showing that these techniques work well also in this scenario. In this paper, we will consider the extension of these multiuser techniques to the case of CPM signals, since they are often employed in satellite communications for their robustness to non-linearities, stemming from the constant envelope, their claimed power and spectral efficiency, and their recursive nature which allows to employ them in serially concatenated schemes [4], [5].

The literature on MUD for FDM-CPM systems is very poor and essentially focuses on binary schemes, such as Gaussian minimum shift keying (GMSK). In [6], the theoretical limits of a multiuser communication system using serially concatenated CPMs (SCCPMs) over an additive white Gaussian noise (AWGN) channel are investigated. Reduced-complexity MUD techniques are not considered in [6], but it is suggested that most of the methods proposed for CDMA could be applied to multiuser SCCPM schemes.

Reduced-complexity MUD techniques based on soft interference cancellation (SIC) (e.g., see [7], [8]) from the CDMA literature have been derived relying on a Gaussian approximation of the multiple access interference (MAI). In this paper, these suboptimal MUD schemes will be extended to the FDM-CPM scenario. We will also propose a new scheme using the framework based on factor graphs (FGs) and the sum-product algorithm (SPA) [9].

After the system model description of Section II, in Section III optimal and suboptimal reduced-complexity MUD schemes are extended to the FDM-CPM scenario and a new algorithm is also proposed. This new scheme is obtained by means of some graphical manipulations on the FG representing the joint a posteriori probability mass function (pmf) of the transmitted symbols, rather than assuming the interference as Gaussian, as suggested in [7], [8], [10]. Possible extensions of the new scheme are also discussed. The performance/complexity analysis is reported in Section IV and finally some conclusions are drawn in Section V. The proposed approach can be also employed to derive new detection schemes for other scenarios, such as CDMA systems [11] or spectrally-efficient FDM systems based on linear modulations.

\section{System Model}

We assume that the channel is shared by $U$ independent users. Without loss of generality, we consider synchronous users and an AWGN channel. The extension to the case of asynchronous users will be discussed later.

We assume that each user transmits $N$ symbols and we denote by $\alpha_{n}^{(u)}$ the symbol transmitted by user $u$ at 
discrete-time $n$, which takes on values in the $M^{(u)}$-ary alphabet $\left\{ \pm 1, \pm 3 \cdots \pm\left(M^{(u)}-1\right)\right\}$. Moreover, $\boldsymbol{\alpha}^{(u)}=$ $\left(\alpha_{0}^{(u)}, \ldots, \alpha_{N-1}^{(u)}\right)^{T}$ is the vector of the $N$ symbols transmitted by user $u$ and we also denote $\boldsymbol{\alpha}_{n}=\left(\alpha_{n}^{(1)}, \ldots, \alpha_{n}^{(U)}\right)^{T}$ and $\boldsymbol{\alpha}=\left(\boldsymbol{\alpha}_{0}^{T}, \ldots, \boldsymbol{\alpha}_{N-1}^{T}\right)^{T}{ }^{2}$ The complex envelope of the received signal can be written as

$$
r(t)=\sum_{u=1}^{U} s^{(u)}\left(t, \boldsymbol{\alpha}^{(u)}\right) \exp \left\{j 2 \pi f^{(u)} t\right\}+w(t)
$$

where $w(t)$ is a zero-mean circularly symmetric white Gaussian noise process with power spectral density $2 N_{0}\left(N_{0}\right.$ is assumed perfectly known at the receiver), $f^{(u)}$ is the difference between the carrier frequency of user $u$ and the frequency assumed as reference for the computation of the complex envelope, and $s^{(u)}\left(t, \boldsymbol{\alpha}^{(u)}\right)$ is the CPM information-bearing signal of user $u$ which reads

$s^{(u)}\left(t, \boldsymbol{\alpha}^{(u)}\right)=\sqrt{\frac{2 E_{S}^{(u)}}{T}} \exp \left\{j 2 \pi h^{(u)} \sum_{n=0}^{N-1} \alpha_{n}^{(u)} q^{(u)}(t-n T)\right\}$.

In (2), $E_{S}^{(u)}$ is the energy per information symbol of user $u, T$ the symbol interval, common to all users, $q^{(u)}(t)$ the phase-smoothing response, and $h^{(u)}=r^{(u)} / p^{(u)}$ the modulation index $\left(r^{(u)}\right.$ and $p^{(u)}$ are relatively prime integers). The derivative of the function $q^{(u)}(t)$ is the so-called frequency pulse of length $L^{(u)}$ symbol intervals. In the generic time interval $[n T, n T+T)$, the CPM signal of user $u$ is completely defined by symbol $\alpha_{n}^{(u)}$ and state $\sigma_{n}^{(u)}=\left(\omega_{n}^{(u)}, \phi_{n}^{(u)}\right)$ [12], where

$$
\omega_{n}^{(u)}=\left(\alpha_{n-1}^{(u)}, \alpha_{n-2}^{(u)}, \ldots, \alpha_{n-L^{(u)}+1}^{(u)}\right)
$$

is the correlative state and $\phi_{n}^{(u)}$ is the phase state which can be recursively defined as

$$
\phi_{n}^{(u)}=\left[\phi_{n-1}^{(u)}+\pi h^{(u)} \alpha_{n-L^{(u)}}^{(u)}\right]_{2 \pi}
$$

where $[\cdot]_{2 \pi}$ denotes the "modulo $2 \pi$ " operator, and takes on $p^{(u)}$ values. In the following, we define $\sigma_{n}=$ $\left(\sigma_{n}^{(1)}, \ldots, \sigma_{n}^{(U)}\right)^{T}$ and $\boldsymbol{\sigma}=\left(\boldsymbol{\sigma}_{0}^{T}, \ldots, \boldsymbol{\sigma}_{N}^{T}\right)^{T}$.

Considering the useful component of the received signal (1), in the time interval $[n T, n T+T)$ only $\prod_{u=1}^{U} p^{(u)} M^{(u) L^{(u)}}$ possible waveforms are allowed. Hence, a set of sufficient statistics for detection can be obtained through projection of each slice of duration $T$ of the received signal $r(t)$ onto an orthonormal basis, of cardinality at most $\prod_{u=1}^{U} M^{(u) L^{(u)}}$, of the signal space of these possible waveforms. The use of an orthonormal basis will ensure that the noise components are independent and identically distributed (i.i.d.) complex Gaussian random variables with independent components, each with mean zero and variance $N_{0}$. In practical receivers, an approximated set of sufficient statistics is obtained through the technique described in [13]. It is assumed that the useful signal component in $r(t)$ is band-limited-although this is not strictly true in the case of CPM signals, whose spectrum has an infinite support-with bandwidth lower than $\eta / 2 T$, where $\eta$ is a proper integer. The approximated statistics can be obtained by extracting $\eta$ samples per symbol interval from

${ }^{2}$ In the following, $(\cdot)^{T}$ denotes transpose and $(\cdot)^{H}$ transpose conjugate. the received signal prefiltered by means of an analog lowpass filter which leaves unmodified the useful signal and has a vestigial symmetry around $\eta / 2 T .^{3}$ The condition on the vestigial symmetry of the analog prefilter ensures that the noise samples are i.i.d. complex Gaussian random variables with independent components, each with mean zero and variance $\Xi^{2}=N_{0} \eta / T$.

Although not necessary in the derivation of the algorithms, since it applies unmodified independently of the employed orthogonal basis, the only difference being the noise variance, in the numerical results we will assume a sufficient statistic obtained through oversampling. We will denote by $r_{n, m}$ the $m$-th received sample $(m=0,1, \ldots, \eta-1)$ of the $n$-th symbol interval. It can be expressed as

$$
r_{n, m}=\sum_{u=1}^{U} s_{n, m}^{(u)}\left(\alpha_{n}^{(u)}, \sigma_{n}^{(u)}\right)+w_{n, m}
$$

where, as mentioned, $\left\{w_{n, m}\right\}$ are independent and identically distributed complex Gaussian noise samples and $s_{n, m}^{(u)}\left(\alpha^{(u)}, \sigma^{(u)}\right)$ (whose dependence on $\alpha^{(u)}$ and $\sigma^{(u)}$ will be omitted in the following) is the contribution of user $u$ to the useful signal component. In the following, we will define $\mathbf{r}_{n}=\left(r_{n, 0}, r_{n, 1}, \ldots, r_{n, \eta-1}\right)^{T}, \mathbf{r}=\left(\mathbf{r}_{0}^{T}, \mathbf{r}_{1}^{T}, \ldots, \mathbf{r}_{N-1}^{T}\right)^{T}$ and $\mathbf{s}_{n}^{(u)}=\left(s_{n, 0}^{(u)}, s_{n, 1}^{(u)}, \ldots, s_{n, \eta-1}^{(u)}\right)^{T}$.

\section{Multiuser ReCEIVERS FOR FDM-CPM Systems}

We now derive several soft-input soft-output (SISO) MUD algorithms for FDM-CPM systems.

\section{A. Optimal multiuser detector}

From (5) and assuming that symbols of user $u$ are independent, the pmf $P(\boldsymbol{\alpha}, \boldsymbol{\sigma} \mid \mathbf{r})$ factorizes as ${ }^{4}$

$$
P(\boldsymbol{\alpha}, \boldsymbol{\sigma} \mid \mathbf{r}) \propto p(\mathbf{r} \mid \boldsymbol{\alpha}, \boldsymbol{\sigma}) P(\boldsymbol{\sigma} \mid \boldsymbol{\alpha}) P(\boldsymbol{\alpha})
$$

where

$$
\begin{aligned}
P(\boldsymbol{\alpha}) & =\prod_{n=0}^{N-1} P\left(\boldsymbol{\alpha}_{n}\right) \\
P(\boldsymbol{\sigma} \mid \boldsymbol{\alpha}) & =P\left(\boldsymbol{\sigma}_{0}\right) \prod_{n=0}^{N-1} P\left(\boldsymbol{\sigma}_{n+1} \mid \boldsymbol{\sigma}_{n}, \boldsymbol{\alpha}_{n}\right) \\
p(\mathbf{r} \mid \boldsymbol{\alpha}, \boldsymbol{\sigma}) & \propto \prod_{n=0}^{N-1} \exp \left\{-\frac{1}{2 \Xi^{2}}\left\|\mathbf{r}_{n}-\sum_{u=1}^{U} \mathbf{s}_{n}^{(u)}\right\|^{2}\right\} .
\end{aligned}
$$

Notice that $P\left(\boldsymbol{\sigma}_{n+1} \mid \boldsymbol{\sigma}_{n}, \boldsymbol{\alpha}_{n}\right)$ is an indicator function, equal to one if $\boldsymbol{\alpha}_{n}, \boldsymbol{\sigma}_{n}$, and $\boldsymbol{\sigma}_{n+1}$ satisfy the trellis constraints of each user and to zero otherwise. Defining

$$
\begin{aligned}
M_{n}\left(\boldsymbol{\alpha}_{n}, \boldsymbol{\sigma}_{n}, \boldsymbol{\sigma}_{n+1}\right)= & P\left(\boldsymbol{\sigma}_{n+1} \mid \boldsymbol{\sigma}_{n}, \boldsymbol{\alpha}_{n}\right) \\
& \cdot \exp \left\{-\frac{1}{2 \Xi^{2}}\left\|\mathbf{r}_{n}-\sum_{u=1}^{U} \mathbf{s}_{n}^{(u)}\right\|^{2}\right\}
\end{aligned}
$$

\footnotetext{
${ }^{3}$ This approach is equivalent of using an orthogonal basis of properly delayed sinc functions.

${ }^{4}$ In the following, $P(\cdot)$ [respectively, $p(\cdot)$ ] denotes the pmf [resp., the probability density function (pdf)] of a discrete (resp., continuous) random vector.
} 


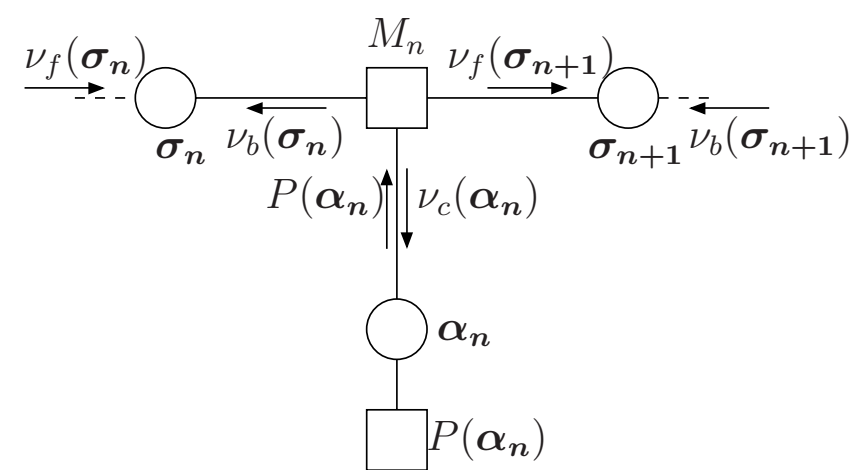

Fig. 1. FG related to (9).

we now have

$$
P(\boldsymbol{\alpha}, \boldsymbol{\sigma} \mid \mathbf{r}) \propto P\left(\boldsymbol{\sigma}_{0}\right) \prod_{n=0}^{N-1} M_{n}\left(\boldsymbol{\alpha}_{n}, \boldsymbol{\sigma}_{n}, \boldsymbol{\sigma}_{n+1}\right) P\left(\boldsymbol{\alpha}_{n}\right)
$$

whose corresponding FG is shown in Fig. 1. The SPA applied to this cycle-free FG takes the form of a forward/backward algorithm [9] (cf. Fig. 1 with [9, Fig. 14]), also known as BCJR algorithm [14], and provides the exact a posteriori probabilities $P\left(\alpha_{n}^{(u)} \mid \mathbf{r}\right)$. We recall here the main ideas for a reader not familiar with the FG/SPA framework. Nodes in the graph ideally become elementary processors which, according to the SPA rules, compute and exchange messages along communication channels represented by graph edges [9]. With reference to the messages represented in Fig. 1, by applying the SPA rules [9] we obtain the following forward and backward recursions

$$
\begin{aligned}
\nu_{f}\left(\boldsymbol{\sigma}_{n+1}\right) & =\sum_{\boldsymbol{\alpha}_{n}} \sum_{\boldsymbol{\sigma}_{n}} M_{n}\left(\boldsymbol{\alpha}_{n}, \boldsymbol{\sigma}_{n}, \boldsymbol{\sigma}_{n+1}\right) \nu_{f}\left(\boldsymbol{\sigma}_{n}\right) P\left(\boldsymbol{\alpha}_{n}\right) \\
\nu_{b}\left(\boldsymbol{\sigma}_{n}\right) & =\sum_{\boldsymbol{\alpha}_{n}} \sum_{\boldsymbol{\sigma}_{n+1}} M_{n}\left(\boldsymbol{\alpha}_{n}, \boldsymbol{\sigma}_{n}, \boldsymbol{\sigma}_{n+1}\right) \nu_{b}\left(\boldsymbol{\sigma}_{n+1}\right) P\left(\boldsymbol{\alpha}_{n}\right)
\end{aligned}
$$

with proper initial conditions [9], and the following final completion to compute the a posteriori probabilities

$$
\begin{aligned}
& P\left(\alpha_{n}^{(u)} \mid \mathbf{r}\right)=P\left(\alpha_{n}^{(u)}\right) \nu_{c}\left(\alpha_{n}^{(u)}\right) \\
& =P\left(\alpha_{n}^{(u)}\right) \sum_{\boldsymbol{\sigma}_{n}} \sum_{\boldsymbol{\sigma}_{n+1}} M_{n}\left(\boldsymbol{\alpha}_{n}, \boldsymbol{\sigma}_{n}, \boldsymbol{\sigma}_{n+1}\right) \nu_{f}\left(\boldsymbol{\sigma}_{n}\right) \nu_{b}\left(\boldsymbol{\sigma}_{n+1}\right) .
\end{aligned}
$$

The complexity of this optimal multiuser detector is exponential in the number of users.

\section{B. SIC algorithms from the CDMA literature}

The most efficient reduced-complexity SIC algorithm from the CDMA literature is that proposed in [7], [8] (see also [10]). It is based on a Gaussian approximation for the MAI. In other words, the algorithm can be obtained by replacing the pmf of the interfering symbols with a complex circularly symmetric Gaussian pdf with the same mean and variance. A SISO detector for each user will be employed and they will exchange soft information used to cancel out the interference.

In order to generalize the algorithm to the considered scenario, we assume that the equivalent channel for user $i$ is

$$
r_{n, m}^{(i)}=s_{n, m}^{(i)}+z_{n, m}^{(i)}
$$

where $z_{n, m}^{(i)}$ is the sum of interference and noise, given by

$$
z_{n, m}^{(i)}=w_{n, m}+\sum_{u=1, u \neq i}^{U} s_{n, m}^{(u)}
$$

The vector $\mathbf{z}_{n}^{(i)}=\left(z_{n, 0}^{(i)}, z_{n, 1}^{(i)} \ldots, z_{n, \eta-1}^{(i)}\right)^{T}$, assumed to be Gaussian, has mean $\boldsymbol{\mu}_{n}^{(i)}$ and covariance matrix $\boldsymbol{\Phi}_{n}^{(i)}$ given by

$$
\begin{aligned}
\boldsymbol{\mu}_{n}^{(i)}= & \sum_{u=1, u \neq i}^{U} \sum_{\left(\alpha_{n}^{(u)}, \sigma_{n}^{(u)}\right)} \hat{P}\left(\alpha_{n}^{(u)}, \sigma_{n}^{(u)} \mid \mathbf{r}\right) \mathbf{s}_{n}^{(u)} \\
\boldsymbol{\Phi}_{n}^{(i)}= & \sum_{u=1, u \neq i}^{U} \sum_{\left(\alpha_{n}^{(u)}, \sigma_{n}^{(u)}\right)} \hat{P}\left(\alpha_{n}^{(u)}, \sigma_{n}^{(u)} \mid \mathbf{r}\right) \mathbf{s}_{n}^{(u)} \mathbf{s}_{n}^{(u) H} \\
& -\boldsymbol{\mu}_{n}^{(i)} \boldsymbol{\mu}_{n}^{(i) H}+2 \Xi^{2} \mathbf{I}
\end{aligned}
$$

where $\mathbf{I}$ is the identity matrix and $\hat{P}\left(\alpha_{n}^{(u)}, \sigma_{n}^{(u)} \mid \mathbf{r}\right)$ are the estimates of the a posteriori probabilities provided by the SISO detector related to the interfering user $u$. The SISO detector for user $i$, in the form of a BCJR algorithm, will employ Gaussian branch metrics computed from $\boldsymbol{\mu}_{n}^{(i)}$ and $\boldsymbol{\Phi}_{n}^{(i)}$ [7], [8], [10]. This suboptimal multiuser detector is then composed of $U$ single-user detectors whose complexity is increased, with respect to the case when the interference is neglected, by the need to compute, for each symbol interval, $\boldsymbol{\mu}_{n}^{(i)}$ and $\boldsymbol{\Phi}_{n}^{(i)}$, through (10) and (11), to perform the inversion of $\boldsymbol{\Phi}_{n}^{(i)}$, and to finally compute the quadratic form in the branch metrics. In the following, this algorithm will be referred to as SIC 1.

The algorithm can be simplified by neglecting the offdiagonal elements of $\boldsymbol{\Phi}_{n}^{(i)}$ [7]. In this way, the inversion results to be computationally less intensive at the price of a performance degradation. This simplified detector will be referred to as SIC 2.

\section{Proposed algorithm}

By using the FG/SPA framework, we now derive a new algorithm without resorting to a Gaussian approximation of the MAI. The algorithm results from a different factorization of the pmf $P(\boldsymbol{\alpha}, \boldsymbol{\sigma} \mid \mathbf{r})$. In fact we can further factorize (6), (7), and (8) as

$$
\begin{aligned}
P(\boldsymbol{\alpha}) & =\prod_{u=1}^{U} \prod_{n=0}^{N-1} P\left(\alpha_{n}^{(u)}\right) \\
P(\boldsymbol{\sigma} \mid \boldsymbol{\alpha}) & =\prod_{u=1}^{U} P\left(\sigma_{0}^{(u)}\right) \prod_{n=0}^{N-1} P\left(\sigma_{n+1}^{(u)} \mid \sigma_{n}^{(u)}, \alpha_{n}^{(u)}\right) \\
& =\prod_{u=1}^{U} P\left(\sigma_{0}^{(u)}\right) \prod_{n=0}^{N-1} I_{n}^{(u)}\left(\alpha_{n}^{(u)}, \sigma_{n}^{(u)}, \sigma_{n+1}^{(u)}\right) \\
p(\mathbf{r} \mid \boldsymbol{\alpha}, \boldsymbol{\sigma}) & \propto \prod_{n=0}^{N-1} F_{n}\left(\boldsymbol{\alpha}_{n}, \boldsymbol{\sigma}_{n}\right) \prod_{u=1}^{U} H_{n}^{(u)}\left(\alpha_{n}^{(u)}, \sigma_{n}^{(u)}\right)
\end{aligned}
$$




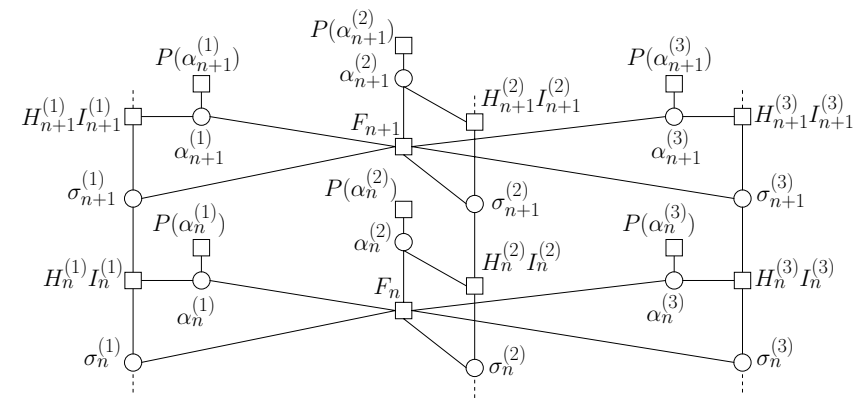

Fig. 2. FG corresponding to (15) for $U=3$.

where

$$
\begin{aligned}
& I_{n}^{(u)}\left(\alpha_{n}^{(u)}, \sigma_{n}^{(u)}, \sigma_{n+1}^{(u)}\right)=P\left(\sigma_{n+1}^{(u)} \mid \sigma_{n}^{(u)}, \alpha_{n}^{(u)}\right) \\
& H_{n}^{(u)}\left(\alpha_{n}^{(u)}, \sigma_{n}^{(u)}\right)=\exp \left\{\frac{1}{\Xi^{2}} \operatorname{Re}\left[\mathbf{r}_{n}^{H} \mathbf{s}_{n}^{(u)}\right]\right\} \\
& F_{n}\left(\boldsymbol{\alpha}_{n}, \boldsymbol{\sigma}_{n}\right)=\prod_{i=1}^{U-1} \prod_{j=i+1}^{U} \exp \left\{-\frac{1}{\Xi^{2}} \operatorname{Re}\left[\mathbf{s}_{n}^{(i) H} \mathbf{s}_{n}^{(j)}\right]\right\}
\end{aligned}
$$

having discarded the terms independent of symbols and states and taken into account that a CPM signal has a constant envelope. Hence, we finally have

$$
\begin{aligned}
& P(\boldsymbol{\alpha}, \boldsymbol{\sigma} \mid \mathbf{r}) \propto\left[\prod_{u=1}^{U} P\left(\sigma_{0}^{(u)}\right)\right] \prod_{n=0}^{N-1} F_{n}\left(\boldsymbol{\alpha}_{n}, \boldsymbol{\sigma}_{n}\right) \\
& \cdot \prod_{u=1}^{U} H_{n}^{(u)}\left(\alpha_{n}^{(u)}, \sigma_{n}^{(u)}\right) I_{n}^{(u)}\left(\alpha_{n}^{(u)}, \sigma_{n}^{(u)}, \sigma_{n+1}^{(u)}\right) P\left(\alpha_{n}^{(u)}\right) .
\end{aligned}
$$

The resulting graph, shown in Fig. 2, has cycles of length four, that make unlikely the convergence of the SPA, since they are too short. We can remove these short cycles in the original graph by stretching [9] the variables $\sigma_{n}^{(u)}$ in $\left(\alpha_{n}^{(u)}, \sigma_{n}^{(u)}\right)$. In other words, instead of representing variable $\alpha_{n}^{(u)}$ alone, we define a new variable given by the couple $\left(\alpha_{n}^{(u)}, \sigma_{n}^{(u)}\right)$, thus allowing to remove the edges connecting node $F_{n}$ with variable nodes $\sigma_{n}^{(u)}$ [9]. We remark here that this transformation does not involve approximations-the resulting graph still preserves all the information of the original graph. This is not the only graph transformation that we can adopt to remove short cycles. Clustering [9] is the other alternative producing an algorithm with similar performance but slightly greater complexity.

The FG corresponding to this operation has shortest cycles of length twelve and is depicted in Fig. 3 in the case of a system with three users. Obviously, since cycle are still present, the SPA applied to this graph is iterative and leads to an approximate computation of the a posteriori probabilities $P\left(\alpha_{n}^{(u)} \mid \mathbf{r}\right)$. However, the absence of short cycles allows us to obtain very good approximations, as demonstrated by the excellent performance of the algorithm.

We can introduce a further simplification, assuming that the interference among non-adjacent users is negligible. In other words, we approximate (14) as

$$
F_{n}\left(\boldsymbol{\alpha}_{n}, \boldsymbol{\sigma}_{n}\right) \simeq \prod_{i=1}^{U-1} F_{n}^{(i, i+1)}\left(\alpha_{n}^{(i)}, \sigma_{n}^{(i)}, \alpha_{n}^{(i+1)}, \sigma_{n}^{(i+1)}\right)
$$

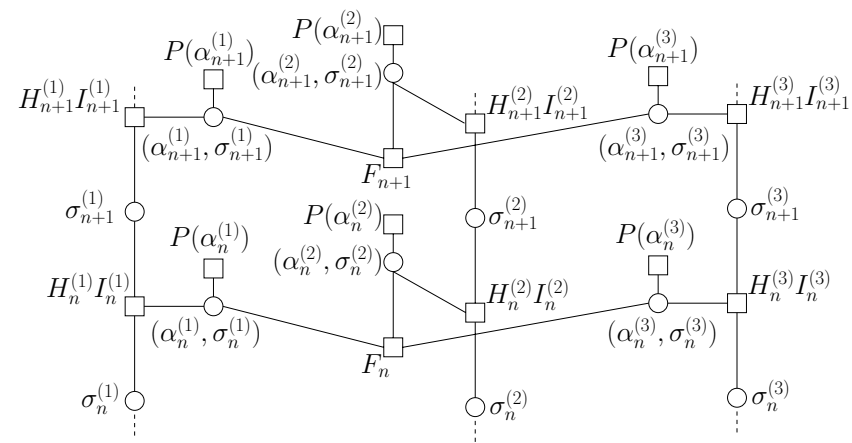

Fig. 3. FG corresponding to (15) after stretching the variables $\sigma_{n}^{(u)}$ in $\left(\alpha_{n}^{(u)}, \sigma_{n}^{(u)}\right)$ and for $U=3$.

where

$$
\begin{aligned}
F_{n}^{(i, i+1)}\left(\alpha_{n}^{(i)}, \sigma_{n}^{(i)},\right. & \left.\alpha_{n}^{(i+1)}, \sigma_{n}^{(i+1)}\right) \\
& =\exp \left\{-\frac{1}{\Xi^{2}} \operatorname{Re}\left[\mathbf{s}_{n}^{(i) H} \mathbf{s}_{n}^{(i+1)}\right]\right\} .
\end{aligned}
$$

The corresponding FG is shown in Fig. 4 for $U=3$. As the reader can observe by comparing Fig. 3 and Fig. 4, this further factorization in (16) implies that node $F_{n}$ is split in two nodes $\left(U-1\right.$ nodes, in general), $F_{n}^{(1,2)}, F_{n}^{(2,3)}$, each of them connecting only two adjacent users.

Let us consider the FG in Fig. 4. If we remove the factor nodes $F_{n}^{(i, i+1)}$, we obtain $U$ single-user detectors which neglect the interference. Hence, these nodes are in charge of the interference mitigation. The increase in complexity due to their presence can be easily understood if we consider their role when we apply the SPA. Let us consider user $i$ and the fact that the messages from node $\left(\alpha_{n}^{(i-1)}, \sigma_{n}^{(i-1)}\right)$ to node $F_{n}^{(i-1, i)}$ and from node $\left(\alpha_{n}^{(i+1)}, \sigma_{n}^{(i+1)}\right)$ to node $F_{n}^{(i, i+1)}$ have the meaning of estimates (approximations) of the a posteriori probabilities $P\left(\alpha_{n}^{(i-1)}, \sigma_{n}^{(i-1)} \mid \mathbf{r}\right)$ and $P\left(\alpha_{n}^{(i+1)}, \sigma_{n}^{(i+1)} \mid \mathbf{r}\right)$, respectively. Hence, the detector for user $i$ will employ as branch metric

$$
\begin{array}{r}
H_{n}^{(i)} I_{n}^{(i)} P\left(\alpha_{n}^{(i)}\right) \sum_{\left(\alpha_{n}^{(i-1)}, \sigma_{n}^{(i-1)}\right)} \hat{P}\left(\alpha_{n}^{(i-1)}, \sigma_{n}^{(i-1)} \mid \mathbf{r}\right) F_{n}^{(i-1, i)} \\
\cdot \sum_{\left(\alpha_{n}^{(i+1)}, \sigma_{n}^{(i+1)}\right)} \hat{P}\left(\alpha_{n}^{(i+1)}, \sigma_{n}^{(i+1)} \mid \mathbf{r}\right) F_{n}^{(i, i+1)} .
\end{array}
$$

For each interfering user and couple $\left(\alpha_{n}^{(i)}, \sigma_{n}^{(i)}\right)$, the number of terms to be summed, which is related to the computational complexity, is identical to that necessary to obtain $\boldsymbol{\mu}_{n}^{(i)}$ or $\boldsymbol{\Phi}_{n}^{(i)}$, through (10) or (11). However, overall, the complexity is much lower than that of SIC 1 since there is no need to compute a matrix inversion and to filter again the received vector for each iteration. The computational aspects of the described algorithms will be extensively discussed in Section IV.

As we will see, this new detector has a better performance than SIC 1 and SIC 2 schemes for the following reason. Since we are considering a FDM scenario, even if we increase the total number of users, there are in practice only a couple of adjacent users (or at most 4 ) that interfere with the considered user. As a consequence, the central limit theory and the Gaussian approximation used to derive SIC 1 and SIC 2 schemes could not be advocated. 


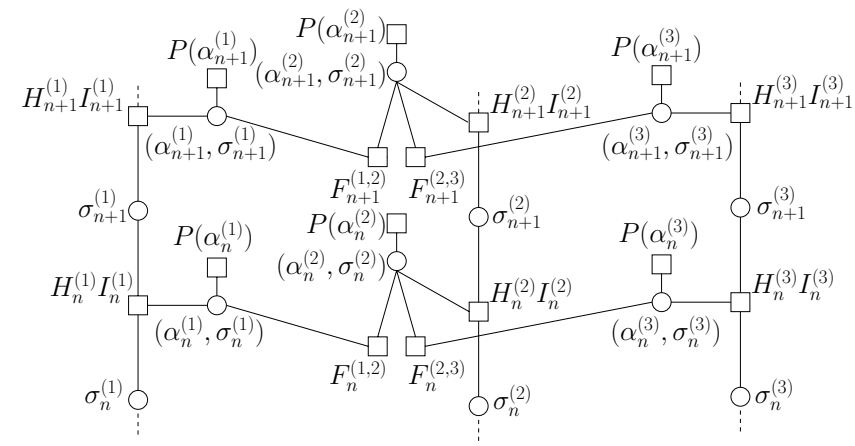

Fig. 4. FG resulting from the approximation (16) and for $U=3$.

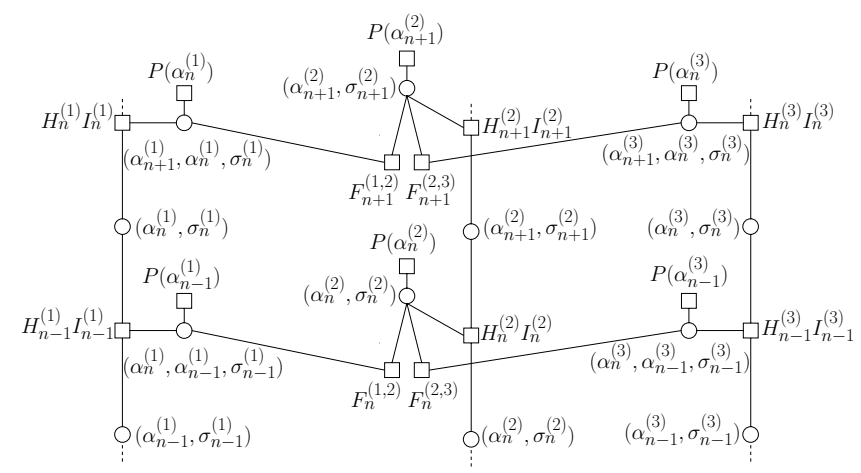

Fig. 5. FG for three asynchronous users.

The continuous-time model in (1) can be easily modified to account for asynchronous users by including their relative delays. The complex envelope of the received signal is now expressed by

$r(t)=\sum_{u=1}^{U} s^{(u)}\left(t-\tau^{(u)}, \boldsymbol{\alpha}^{(u)}\right) \exp \left\{j 2 \pi f^{(u)}\left(t-\tau^{(u)}\right)\right\}+w(t)$

where $\tau^{(u)} \in[0, T)$ is the relative time offset of user $u$, assumed known at the receiver. Without loss of generality, the smallest of these delays can be assumed to be zero. The extension of the proposed algorithm to the more general case of asynchronous users is based on the observation that, in this case, the signals of different users are not time-aligned and the interference mitigation stage involves CPM signals on two successive symbol intervals. In other words, while in the synchronous case the interference mitigation can focus on one symbol interval, the nodes $F_{n}^{(i, j)}$ that connect the pair of users $(i, j)$ in the FG now depend on symbols and states of two consecutive symbol intervals. An example of FG for a system with three asynchronous users with $\tau^{(2)}=0$ is depicted in Fig. 5, showing how to stretch information symbols. It has been obtained neglecting the interference among non-adjacent channels.

Laurent decomposition [15] allows to exactly express a CPM signal as the superposition of $(M-1) 2^{(L-1) \log _{2} M}$ linearly modulated components. Most of the signal power is concentrated in the so-called $M-1$ principal components. Hence, reduced-complexity receivers can be designed starting from an approximation of a CPM signal based on these components only [16], [17]. The proposed MUD algorithm can be redesigned based on an approximate CPM representation using the principal components. The details are omitted for a lack of space. In Section IV, we will show that this approach allows to further reduce the computational complexity with a limited performance degradation.

\section{Numerical Results}

In this section, we report a performance/complexity comparison for the described reduced-complexity MUD algorithms, the optimal multiuser detector (O-MUD), and the single-user detector (SUD) which simply neglects the interference. The O-MUD curve will be a lower bound on the performance achieved by any low-complexity algorithm. In the following, we will assume that all users adopt the same modulation format. Hence, in the expression of the alphabet cardinality $M^{(u)}$, modulation index $h^{(u)}$, number of phase states $p^{(u)}$, and length of the frequency pulse $L^{(u)}$, superscript will be omitted.

\section{A. Complexity considerations}

The computational complexity is assessed with reference to the coherent synchronous case. We assume that the computation of a non linear function is performed by using a lookup table (LUT). The number of additions between two real arguments and accesses to LUT of the considered algorithms, per user, per symbol, and per iteration, is reported in Table I and refers to a logarithmic-domain implementation, which is known to provide better numerical stability and to reduce the computational complexity [18]. The operations performed at the first iterations only have been neglected. For the described reduced-complexity MUD algorithms, i.e., SIC 1, SIC 2, and the proposed one, we did not exploit the following trick to further reduce the complexity. For illustration purposes, let us consider the proposed algorithm. As mentioned, it is composed by $U$ SUDs plus the interference mitigation related to the presence of some additional nodes. When only the interference among adjacent users is accounted for and considering user $u$, in order to compute the function $H_{n}^{(u)}$ in (13) and functions $F_{n}^{(u-1, u)}$ and $F_{n}^{(u, u+1)}$ in (17), a reduced sampling frequency can be adopted, provided that the received signal is properly prefiltered, computed assuming that only users $u-1, u$, and $u+1$, are present. In this case, the number of samples per symbols, and hence the computational complexity, is reduced at the expense of an increased number of prefilters-one prefilter and a different set of samples must be used for each user.

In evaluating the complexity of SIC 1 , we have considered the recursive implementation proposed in [7] for the required matrix inversion. Even with this optimization, the matrix inversion represents one of the bottlenecks of the algorithm from a complexity viewpoint. Note that the proposed algorithm, using or not the approximate Laurent decomposition, is characterized by a complexity which increases linearly with the number of users, like SIC 2, whereas the O-MUD algorithm has a complexity which grows exponentially with $U$. Regarding SIC 1, its complexity seems to increase linearly with $U$. However, it increases quadratically with $\eta$, which in turns depends on the number of interfering users. 
TABLE I

COMPUTATIONAL LOAD PER USER, PER SYMBOL, PER ITERATION.

\begin{tabular}{|c|c|c|}
\hline Algorithm & Additions & LUT accesses \\
\hline \hline Proposed & $4\left(p M^{L}\right)^{2}(U-1)+p M^{L}(2 U+13)$ & $\left(p M^{L}\right)^{2}(U-1)+p M^{L}(4-U)$ \\
& $-3 p M^{L-1}-3 M$ & $-p M^{L-1}-M$ \\
\hline Proposed LAU & $p M(3 p M-1)(2 L+1)(U-1)$ & $p M(p M-1)(2 L+1)(U-1)$ \\
& $+(p M)^{2}+17 p M-3 p-3 M-3$ & $+3 p M-p-M-1$ \\
\hline SIC 1 & $p M^{L}\left[\eta^{2}(3 U+5)+\eta(15 U-1)+18\right]$ & $p M^{L}\left[\eta^{2}(4 U+8)+\eta(16 U-4)+3\right]$ \\
& $+\eta^{2}(8 U+22)+\eta(4-4 U)-3 p M^{L-1}-3 M$ & $+\eta^{2}(13 U+33)+\eta(2-2 U)-p M^{L-1}-M$ \\
\hline SIC 2 & $p M^{L}[\eta(14 U-10)+18]$ & $p M^{L}[\eta(15 U-2)+3]$ \\
& $+\eta(4-4 U)-3 p M^{L-1}-3 M$ & $+\eta(2-2 U)-p M^{L-1}-M$ \\
\hline O-MUD & {$\left[\left(p M^{L-1}\right)^{U}\left(15 M^{U}-3\right)-3 M^{U}\right] / U-3\left(M^{U}-M\right)$} & {$\left[\left(p M^{L-1}\right)^{U}\left(3 M^{U}-1\right)-M^{U}\right] / U+M^{U}-M$} \\
\hline SUD & $p M^{L-1}(15 M-3)-3 M$ & $p M^{L-1}(3 M-1)-M$ \\
\hline
\end{tabular}

\section{B. Performance}

We consider SCCPM schemes with iterative decoding employing the described multiuser detectors as inner SISO detectors. We assume that all users transmit at the same power, employ, as mentioned, the same modulation format, and that channels are equally spaced in frequency. The normalized spacing will be denoted by $F=\left|f^{(i)}-f^{(i-1)}\right| T$. Each user employs a different randomly-generated bit-interleaver. The bit error rate (BER) performance for the middle user only is shown versus $E_{b} / N_{0}, E_{b}$ being the received signal energy per information bit. We assume that the users are synchronous. However, we also carried out a BER analysis in the asynchronous scenario observing the same performance as for the synchronous case.

For the described suboptimal multiuser detectors, the performance also depends on the adopted schedule. Serial or parallel schedules are usually considered in the literature [10]. For a lack of space, and also because the difference in performance is practically negligible in this scenario of users transmitting at the same power, we only consider the parallel schedule. In this case, at each iteration all users are activated simultaneously. The computed soft-outputs are then provided to the other users for the next iteration and, after deinterleaving, to the decoders. Note that the chosen schedule impacts the latency but not the complexity of the algorithms, so our computational complexity analysis is independent of this implementation aspect.

In Fig 6, we consider the concatenation of an outer nonrecursive rate- $1 / 2$ convolutional code with generators $(5,7)$ (octal notation) and a minimum shift keying (MSK) modulation, i.e., a binary modulation with $h=1 / 2$ and a rectangular frequency pulse of duration $T$ (1-REC). An interleaver of length 2048 bits is used and a maximum of 15 iterations is allowed. We assume that $U=5$ users transmit simultaneously with a normalized spacing $F=0.5$, leading to a spectral efficiency $\gamma=1 \mathrm{~b} / \mathrm{s} / \mathrm{Hz} .^{5}$ We consider an oversampling factor $\eta=7$, since we found no improvement when considering higher values. From Fig. 6 it can be observed that the proposed algorithm achieves the best performance among suboptimal receivers. In particular, at a BER of $10^{-3}$ the proposed algorithm gains about $1.1 \mathrm{~dB}$ with respect to SIC 1 and $1.5 \mathrm{~dB}$ with respect to SIC 2. Moreover, the loss of the proposed

\footnotetext{
${ }^{5}$ The spectral efficiency is defined as $\gamma=\frac{\rho \log _{2} M}{F} \mathrm{~b} / \mathrm{s} / \mathrm{Hz}$, where $\rho$ is the code rate.
}

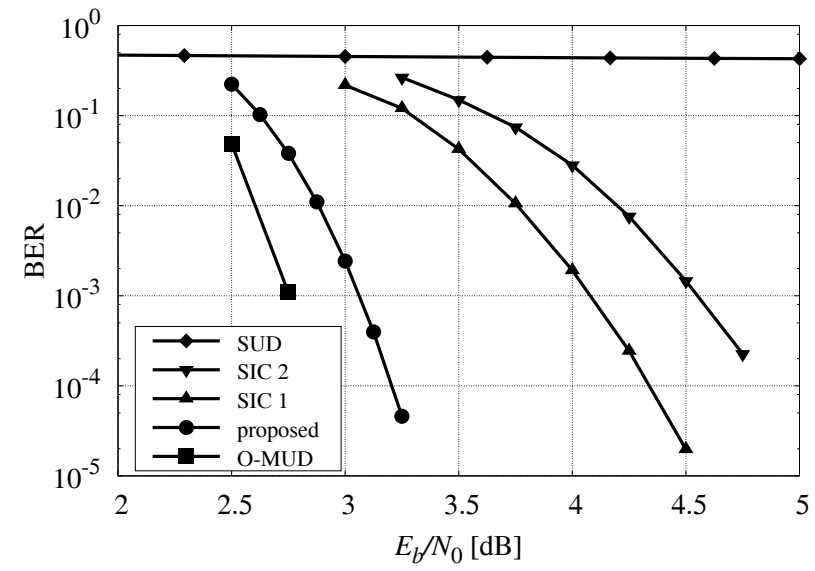

Fig. 6. Non-recursive rate- $1 / 2$ convolutional code with generators $(5,7)$ concatenated (through an interleaver of length 2048 bits) with the MSK modulation. System with $U=5$ and a channel spacing $F=0.5$.

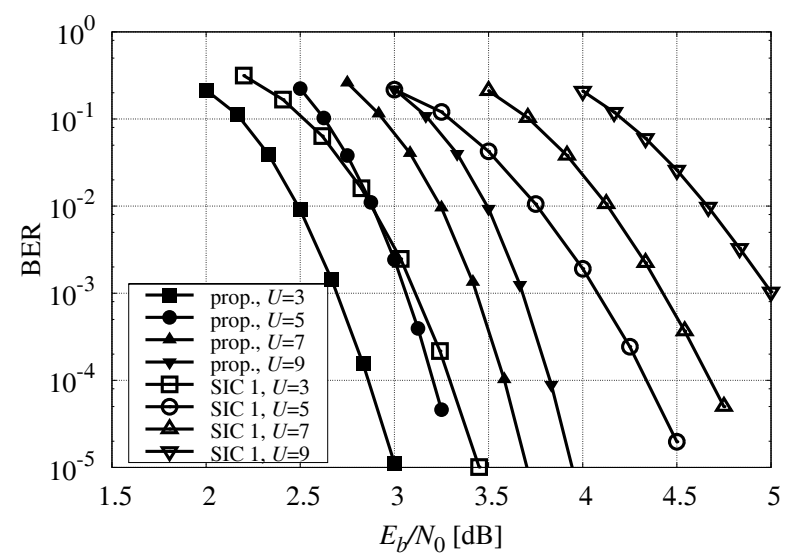

Fig. 7. Non-recursive rate- $1 / 2$ convolutional code with generators $(5,7)$ concatenated (through an interleaver of length 2048 bits) with the MSK modulation. Channel spacing $F=0.5$ and different number of users.

algorithm with respect to the optimal multiuser detector is about $0.3 \mathrm{~dB}$.

The impact on the performance of the number of users is investigated in Fig. 7. In this figure, we assume the same system parameters as in Fig. 6, namely a non-recursive rate$1 / 2$ convolutional code with generators $(5,7)$ concatenated with the MSK modulation and $F=0.5$. Only the SIC 1 
TABLE II

COMPUTATIONAL LOAD PER USER, PER SYMBOL, PER ITERATION FOR THE SCENARIO CONSIDERED IN FIG. 8

\begin{tabular}{|c||c|c|}
\hline \multicolumn{1}{|c||}{} & \multicolumn{2}{c|}{ Fig. 8 } \\
\hline \hline Algorithm & Additions & LUT accesses \\
\hline Proposed & 19296 & 4640 \\
\hline Proposed LAU & 4524 & 1348 \\
\hline SIC 1 & 29286 & 36468 \\
\hline SIC 2 & 8456 & 10428 \\
\hline O-MUD & 550988 & 110055 \\
\hline SUD & 672 & 128 \\
\hline
\end{tabular}

scheme and the proposed algorithm described in Section III-C are considered for $U=3,5,7$, and 9 . As expected, in both cases the performance degrades when the number of users increases - the case of $U=3$ is obviously the most favourable one due to the larger impact of side users which are less subject to interference, can be detected with larger reliability, and whose effect can be better mitigated on the central user of interest. We may observe that the performance advantage of the algorithm in Section III-C increases with $U$. This is a proof of what claimed before-the Gaussian approximation hardly fits the considered scenario.

In Fig. 8 , we consider a quaternary $(M=4) \mathrm{CPM}$ with a raised-cosine (RC) frequency pulse of length $L=2$ (2$\mathrm{RC}), h=1 / 3$, and Gray mapping. The block size for each user is of 1024 information bits and the outer code is a (128, 113) extended Bose, Ray-Chaudhuri, Hocquenghem (eBCH) code with rate $\rho=0.88$ described in [19]. At the receiver side, a maximum of 15 iterations is performed. We consider a system with $U=3$ users, $F=0.8$, which corresponds to a spectral efficiency $\gamma=2.2(\mathrm{~b} / \mathrm{s} / \mathrm{Hz})$, and $\eta=5$. The curve labelled "proposed LAU" refers to the simplified algorithm based on Laurent decomposition. From Fig. 8 it can be observed that also in this scenario the proposed algorithm outperforms the other suboptimal receivers. At a BER of $10^{-3}$, the performance gain is approximately $1.2 \mathrm{~dB}$ and $1.4 \mathrm{~dB}$ with respect to SIC 1 and SIC 2 algorithms, respectively. Moreover the SNR degradation compared with the O-MUD curve is approximately $0.7 \mathrm{~dB}$. The loss of the proposed LAU algorithm with respect to the O-MUD is $1.1 \mathrm{~dB}$. Notice that both in this and in the previous scenario, a SUD, which neglects the interference, is practically useless. In Table II, we show the computational complexity of the considered algorithm in the same scenario of Fig. 8. This table clearly shows that the proposed algorithm has a complexity much lower than that of O-MUD and SIC 1 algorithms. Interestingly, the algorithm based on the approximate Laurent representation leads to a further reduction in complexity at a price of a limited performance degradation.

Those reported are only a subset of the extensive simulations we performed, for various modulation formats, coding schemes, and spacings. These cases were selected since for them it was possible to obtain the performance of the O-MUD algorithm in a reasonable time. However, in all other cases we considered, the proposed algorithms always outperformed SIC 1 and SIC 2 schemes.

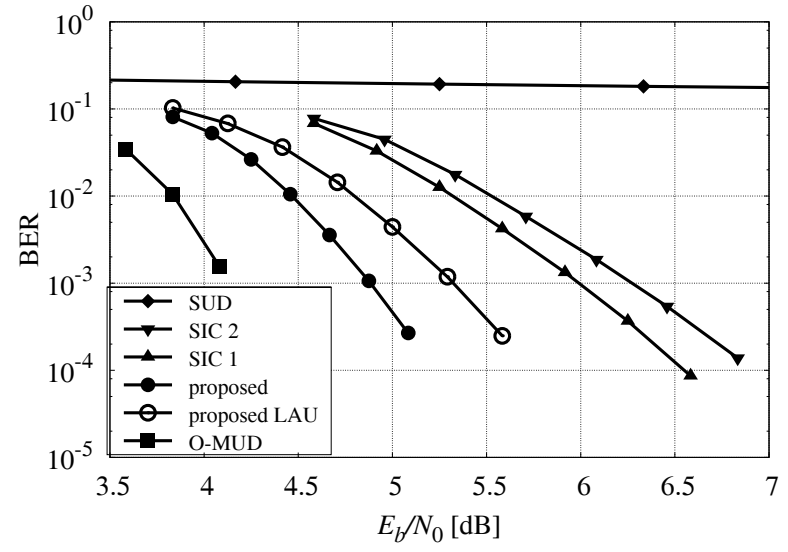

Fig. 8. Outer $(128,113) \mathrm{eBCH}$ code with rate $\rho=0.88$ concatenated with a quaternary $2 \mathrm{RC}$ modulation with $h=1 / 3$. System with $U=3$ and a channel spacing $F=0.8$.

\section{CONCLusions}

In this paper, we discussed the application of reducedcomplexity multiuser detection schemes to frequency division multiplexed systems using continuous phase modulations in serially concatenated schemes. We have extended to this scenario some soft interference cancellation techniques from the CDMA literature and proposed a new detection scheme using the framework based on factor graphs and the sumproduct algorithm. The described algorithms allow to effectively reduce the frequency spacing between adjacent users, thus increasing the spectral efficiency, and in particular, it has been shown that the new proposed suboptimal detection scheme is the most effective in terms of performance and computational complexity.

\section{ACKNOWLEDGMENT}

The authors wish to thank the Editor and the anonymous reviewers for useful comments that improved the presentation of the paper.

\section{REFERENCES}

[1] A. Barbieri, D. Fertonani, and G. Colavolpe, "Time-frequency packing for linear modulations: spectral efficiency and practical detection schemes," IEEE Trans. Commun., vol. 57, pp. 2951-2959, Oct. 2009.

[2] A. Barbieri, D. Fertonani, and G. Colavolpe, "Spectrally-efficient continuous phase modulations," IEEE Trans. Wireless Commun., vol. 8, pp. 1564-1572, Mar. 2009.

[3] B. F. Beidas, H. El Gamal, and S. Kay, "Iterative interference cancellation for high spectral efficiency satellite communications," IEEE Trans. Commun., vol. 50, pp. 31-36, Jan. 2002.

[4] K. R. Narayanan and G. L. Stüber, "Performance of trellis-coded CPM with iterative demodulation and decoding," IEEE Trans. Commun., vol. 49, pp. 676-687, Apr. 2001.

[5] P. Moqvist and T. M. Aulin, "Serially concatenated continuous phase modulation with iterative decoding," IEEE Trans. Commun., vol. 49, pp. 1901-1915, Nov. 2001.

[6] P. Moqvist, "Multiuser serially concatenated continuous phase modulation," Ph.D. thesis, Chalmers University of Technology, Goteborg, Sweden, 2002.

[7] X. Wang and H. V. Poor, "Iterative (turbo) soft interference cancellation and decoding for coded CDMA," IEEE Trans. Commun., vol. 47, pp. 1046-1061, July 1999.

[8] H. El Gamal and E. Geraniotis, "Iterative multiuser detection for coded CDMA signals in AWGN and fading channels," IEEE J. Sel. Areas Commun., vol. 18, pp. 30-41, Jan. 2000. 
[9] F. R. Kschischang, B. J. Frey, and H.-A. Loeliger, "Factor graphs and the sum-product algorithm," IEEE Trans. Inf. Theory, vol. 47, pp. 498-519, Feb. 2001.

[10] J. Boutros and G. Caire, "Iterative multiuser joint decoding: unified framework and asymptotic analysis," IEEE Trans. Inf. Theory, vol. 48, pp. 1772-1793, July 2002.

[11] A. Barbieri, G. Colavolpe, D. Fertonani, and A. Piemontese, "Novel graph-based algorithms for interference cancellation in CDMA systems," in Proc. IEEE Intern. Work. Signal Process. Advances Wireless Commun., Perugia, Italy, pp. 712-716, June 2009.

[12] B. E. Rimoldi, "A decomposition approach to CPM," IEEE Trans. Inf. Theory, vol. 34, pp. 260-270, Mar. 1988.

[13] H. Meyr, M. Oerder, and A. Polydoros, "On sampling rate, analog prefiltering, and sufficient statistics for digital receivers," IEEE Trans. Commun., vol. 42, pp. 3208-3214, Dec. 1994.

[14] L. R. Bahl, J. Cocke, F. Jelinek, and J. Raviv, "Optimal decoding of linear codes for minimizing symbol error rate," IEEE Trans. Inf. Theory, vol. 20, pp. 284-287, Mar. 1974.

[15] U. Mengali and M. Morelli, "Decomposition of $M$-ary CPM signals into PAM waveforms," IEEE Trans. Inf. Theory, vol. 41, pp. 1265-1275, Sept. 1995.

[16] G. Colavolpe and R. Raheli, "Noncoherent sequence detection of continuous phase modulations," IEEE Trans. Commun., vol. 47, pp. 13031307, Sept. 1999.

[17] A. Barbieri and G. Colavolpe, "Simplified soft-output detection of CPM signals over coherent and phase noise channels," IEEE Trans. Wireless Commun., vol. 6, pp. 2486-2496, July 2007.

[18] P. Roberston, E. Villebrun, and P. Hoeher, "Optimal and sub-optimal maximum a posteriori algorithms suitable for turbo decoding," European Trans. Telecommun., vol. 8, pp. 119-125, Mar./Apr. 1997.

[19] A. Graell i Amat, C. A. Nour, and C. Douillard, "Serially concatenated continuous phase modulation for satellite communications," IEEE Trans. Wireless Commun., vol. 8, pp. 3260-3269, June 2009.

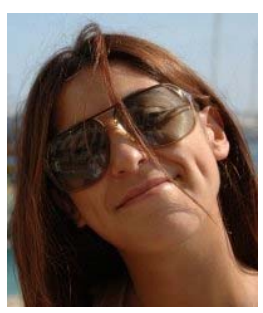

Amina Piemontese (S'09) was born in San Giovanni Rotondo, Italy, in 1980. She received the Dr.Ing. degree in Telecommunications Engineering from the University of Parma, Italy, in April 2006. Currently, she is working toward her Ph.D. degree at the Dipartimento di Ingegneria dell'Informazione, University of Parma, and at the Department of Electronics of TELECOM Bretagne, Brest, France. Her research activity includes various topics in digital communications, with particular emphasis on iterative joint detection and decoding algorithms for

multiuser channels.

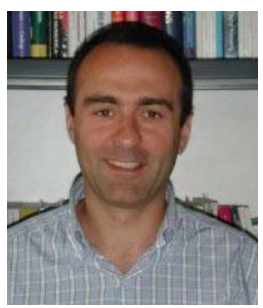

Giulio Colavolpe ( $\left.\mathrm{S}^{\prime} 96-\mathrm{M}^{\prime} 00\right)$ was born in Cosenza, Italy, in 1969. He received the Dr. Ing. degree in Telecommunications Engineering (cum laude) from the University of Pisa, Italy, in 1994 and the Ph.D. degree in Information Technologies from the University of Parma, Italy, in 1998. Since 1997, he has been at the University of Parma, Italy, where he is now an Associate Professor of Telecommunications. In 2000, he was Visiting Scientist at the Institut Eurécom, Valbonne, France.

His main research interests include digital transmission theory, adaptive signal processing, channel coding and information theory. His research activity has led to several scientific publications in leading international journals and conference proceedings and a few industrial patents. He is also co-author of the book Detection Algorithms for Wireless Communications, with Applications to Wired and Storage Systems (New York: John Wiley \& Sons, 2004). He is currently serving as an Editor for the IEEE TransaCtions on Wireless Communications and as an Executive Editor for the European Transactions on Telecommunications.

He received the best paper award at the 13th International Conference on Software, Telecommunications and Computer Networks (SoftCOM'05), Split, Croatia, September 2005 and the best paper award for Optical Networks and Systems at the IEEE International Conference on Communcations (ICC 2008), Beijing, China, May 2008. Dr. Colavolpe is also the principal investigator of several research projects funded by the European Space Agency (ESAESTEC) and important telecommunications companies. 